\title{
Effects on Foot External Rotation of the Modified Ankle-Foot Orthosis on Post-Stroke Hemiparetic Gait
}

\author{
Ha Jeong Kim, MD ${ }^{1}$, Min Ho Chun, $\mathrm{MD}^{2}$, Hong Min Kim, $\mathrm{MD}^{2}$, Bo Ryun Kim, $\mathrm{MD}^{3}$ \\ ${ }^{1}$ Department of Rehabilitation Medicine, Sangju Seongmo Hospital, Sangju; \\ ${ }^{2}$ Department of Rehabilitation Medicine, Asan Medical Center, University of Ulsan College of Medicine, Seoul; \\ ${ }^{3}$ Department of Rehabilitation Medicine, Jeju National University Hospital, Jeju, Korea
}

\begin{abstract}
Objective To evaluate the effects of heel-opened ankle foot orthosis (HOAFO) on hemiparetic gait after stroke, especially on external foot rotation, and to compare the effects of HOAFO with conventional plastic-AFO (pAFO) and barefoot during gait.

Methods This cross-over observational study involved 15 hemiparetic patients with external rotation of the affected foot. All subjects were able to walk independently, regardless of their usual use of a single cane, and had a less than fair-grade in ankle dorsiflexion power. Each patient was asked to walk in three conditions with randomized sequences: 1) barefoot, 2) with a pAFO, and 3) with an HOAFO. Their gait patterns were analyzed using a motion analysis system.

Results Fifteen patients consisted of nine males and six females. On gait analysis, hip and foot external rotation were significantly greater in pAFO $\left(-3.35^{\circ}\right.$ and $\left.-23.68^{\circ}\right)$ than in barefoot and HOAFO conditions $(\mathrm{p}<0.05)$. Wearing an HOAFO resulted in significant decreases in hip $\left(0.78^{\circ}, \mathrm{p}=0.04\right)$ and foot $\left(-17.99^{\circ}, \mathrm{p}<0.01\right)$ external rotation compared with pAFO; although there was no significant difference between HOAFO and barefoot walking. Walking speed and percentage of single limb support were significantly greater for HOAFO than in barefoot walking.

Conclusion HOAFO was superior to pAFO in reducing hip and foot external rotation during the stance phase in patients with post-stroke hemiparesis. HOAFO may, therefore, be useful in patients with excessive external rotation of the foot during conventional pAFO.
\end{abstract}

Keywords Ankle-foot orthosis, Foot rotation, Gait, Stroke

Received November 18, 2012; Accepted April 1, 2013

Corresponding author: Min Ho Chun

Department of Rehabilitation Medicine, Asan Medical Center, University of Ulsan College of Medicine, 88 Olympic-ro 43-gil, Songpa-gu, Seoul 138736, Korea

Tel: +82-2-3010-3800, Fax: +82-2-3010-6964, E-mail: mhchun@amc.seoul.kr

(c) This is an open-access article distributed under the terms of the Creative Commons Attribution Non-Commercial License (http://creativecommons.org/ licenses/by-nc/3.0) which permits unrestricted noncommercial use, distribution, and reproduction in any medium, provided the original work is properly cited. Copyright $\odot 2013$ by Korean Academy of Rehabilitation Medicine 


\section{INTRODUCTION}

Independent ambulation is one of the main goals of stroke rehabilitation. Among the factors that interfere with normal gait in stroke patients are muscle weakness, decreased sensation, impaired coordination, and spasticity of the affected limbs. These post-stroke impairments result in high energy expenditure and abnormal kinetic and kinematic findings, while walking due to forefoot landing, hip circumduction and knee hyperextension [1-3]. Especially, weakness of ankle dorsiflexors usually continues for a long period of time during recovery from a stroke. Among the various methods used to improve ambulation due to ankle weakness, there are also anklefoot orthoses (AFOs), functional electrical stimulation and peroneal nerve stimulation [4-7].

Custom-molded plastic ankle-foot orthoses (pAFOs) are frequently prescribed for hemiparetic patients with stroke. These pAFOs can easily be inserted inside the shoes and prevent feet from dragging during the swing phase of walking and foot inversion in patients with spasticity. In addition, pAFOs have been reported to significantly improve the gait speed, step length, and balance, when compared with that of barefoot gait [8-10].

In hemiparetic gait after stroke, the foot may externally rotate, causing the affected limb to be out in line with the gait direction. This may lead to decreased stability and gait speed [11]. Two studies showed that external rotation increases when wearing pAFO $[12,13]$. The exact mechanism is unknown, but Joo et al. [12] reported that external rotation of the paretic foot results from decreased internal rotation of the hip and increased external rotation of the ankle joint. Although pAFO improves gait speed and stability, correction of this external rotation of the foot may further improve the efficiency of hemiparetic gait with pAFO. Therefore, we have modified a pAFO by opening the calcaneal area, resulting in heel-opened ankle-foot orthosis (HOAFO). Our hypothesis was that HOAFO may reduce external rotation torque by allowing heel cushion and providing sensory feedback, without influencing the ankle stability. We investigate the effects of HOAFO on hemiparetic gait after stroke, especially on external foot rotation during stance phase, and compared the results of HOAFO with those of conventional pAFO and barefoot gait.

\section{MATERIALS AND METHODS}

\section{Participants and clinical measurements}

A total of 15 hemiparetic post-acute stroke patients were recruited from the inpatient rehabilitation department of a tertiary hospital. All of the study subjects had a first onset stroke diagnosed by brain magnetic resonance imaging. Patients were included if they were able to follow a command for more than 2 stages; if they could walk independently regardless of their usual use of a cane; and if they had Modified Ashworth Scale (MAS) 1 to 3 spasticity of the affected ankle and weak ankle dorsiflexion of less than grade 3. Exclusion criteria were severe ankle spasticity or contracture, visual defects or severe hemineglect, unstable medical conditions, or other musculoskeletal or dermatologic problems, such as pressure ulcer in the affected lower limb. The local research ethics committees of our hospital approved the study, and informed consent was obtained from all study subjects.

Patient's age, sex, affected side, and onset duration of stroke were obtained from a review of medical records, and proprioceptive sensory function was measured by physical examination performed by a single physiatrist. We initially measured the impairment or function of the affected limb using several tools. The MAS was used to measure the grade of spasticity in the ankle plantarflexors of the affected side [14]. Motor recovery and function of the affected lower limb were measured using the Brunnstrom stage and Motricity Index (MI), respectively $[15,16]$. The Berg Balance Scale (BBS) was used to assess the balance of subjects [17].

\section{Procedure}

\section{Design of HOAFO}

The conventional pAFO frequently prescribed at our rehabilitation center for stroke patients with spasticity is made of 4 to $4.5 \mathrm{~mm}$ thick polypropylene. The ankle is a $90^{\circ}$ and non-articulated. The length of AFO is about one inch below at the fibular head, with the end of the foot plate located just distal to the metatarsal head. The HOAFO was made of the same material with the same structure, except for cutting the heel area. Part of the heel was cut to expose the calcaneal surface of the rear-foot for sufficient weight-bearing during the stance phase, but was not extended upward to maintain the stability of the ankle joint (Fig. 1). Concretely, it was cut at the half level 


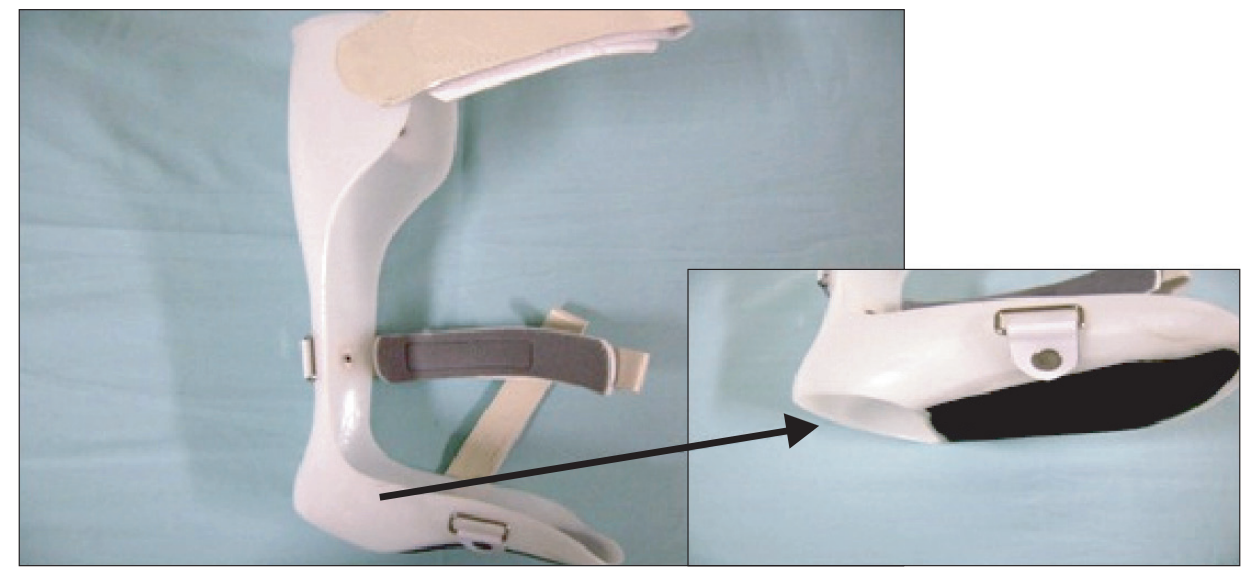

Fig. 1. Left heel-opened ankle foot orthosis. Heel of plastic ankle-foot orthosis was cut to reduce external rotation of foot during stance phase. of calcaneus in an upward direction and transverse tarsal joint area in the front. Three different sizes of pAFO and HOAFO were made, with each subject wearing the orthosis with the best fit. Subjects who already had pAFO or HOAFO wore them during gait analysis.

\section{Gait analysis}

Gait analysis was performed using a motion analysis system (Eagle Digital System; Motion Analysis, Santa Rosa, CA, USA), consisting of six Eagle infrared cameras, two force plates (Advanced Mechanical Technology Inc., Watertown, MA, USA), an Eagle hub (100 Mbps), and an analyzing computer. Spatiotemporal parameters and kinematic data were obtained by the infrared camera, which recognized the markers attached to each subject's body. A total of 19 markers were attached to the subject's lower body segment, including the pelvis, both midthighs, knee joint, mid-point of lower leg, ankle, and foot. Foot markers were placed at the medial and lateral malleolus, mid-point of the heel, and between the second and third metatarsal head. Each subject was asked to walk in three conditions: 1) barefoot, 2) with a pAFO, and 3) with an HOAFO at a self-determined comfortable speed without wearing shoes. The order of the 3 tests was randomized by a block randomization method. The subjects were allowed to walk back and forth five times before recording to familiarize themselves with their orthoses, and they were also allowed to rest for 5 minutes between conditions.

Spatiotemporal parameters included cadence, walking speed, stride length, step width, percentage of single limb support (SLS) and percentage of double limb support (DLS). Kinematic data on the transverse plane were obtained to investigate the external rotation of the pelvis, hip, and foot. The mean angle of rotation during the stance phase was calculated by averaging the maximal angle at three phases of the stance phase: loading response $(0 \%-10 \%)$, midstance $(10 \%-50 \%)$, and terminal stance $(50 \%-60 \%$ of gait cycle). The angle of foot progression was defined as the angle between the line of progression and the line connecting the midpoint of the second and third metatarsal heads and the midpoint of the heel. For convenience, angles of internal rotation were defined as positive (+), and angles of external rotation as negative $(-)$. After gait analysis, each subject was asked about their preference between the two orthoses.

\section{Statistical analysis}

A statistical analysis was performed using SPSS ver. 14.0 (SPSS Inc., Chicago, IL, USA). The gait parameters of the three conditions were compared using repeated measures analysis of the variance (ANOVA). If repeated measures ANOVA showed statistically significant differences between conditions and multiple comparisons (Tukey test) were performed. A p-value $<0.05$ was considered statistically significant.

\section{RESULTS}

Fifteen subjects consisted of nine males and six females, with the mean age of 44.2 years (range, 23-64 years) and average post onset duration of stroke of 57.7 days (range, 25-300 days). Eight subjects had right hemiparesis and seven had left hemiparesis.

All 15 subjects had some spasticity, but not severe on the ankle plantarflexors and their motor function, 
and recovery were generally good on the basis of their Brunnstrom stage, MI, proprioception, and BBS score. Table 1 summarizes the demographic and clinical characteristics of these subjects.

Table 2 shows spatiotemporal parameters of the $3 \mathrm{AFO}$ conditions. Walking speed $(\mathrm{p}<0.01)$ and percentage SLS $(p=0.04)$ were significantly higher with HOAFO than with

Table 1. Demographic and clinical characteristics of study subjects $(\mathrm{n}=15)$

\begin{tabular}{lc}
\hline \multicolumn{1}{c}{ Characteristic } & Value \\
\hline Age (yr) & $44.2 \pm 14.7$ \\
Gender (male:female) & $9: 6$ \\
Affected side of stroke (right:left) & $8: 7$ \\
\hline Stroke onset (day) & $57.7 \pm 70.4$ \\
Modified Ashworth Scale & $0.7 \pm 0.7$ \\
Brunnstrom stage & $4.5 \pm 0.5$ \\
\hline Motricity Index of affected limb & $63.4 \pm 8.8$ \\
Impaired proprioception & 8 \\
\hline Berg Balance Score & $45.1 \pm 6.8$ \\
\hline
\end{tabular}

Values are presented as mean \pm standard deviation. barefoot walking. In contrast, cadence, stride length, step width, and percentage DLS did not differ significantly among the three walking conditions.

Kinematic data of pelvic rotation angle on the transverse plane also did not differ significantly among the three conditions. During barefoot and HOAFO walking in the stance phase, the hip rotation angles were $1.38^{\circ}$ and $0.78^{\circ}$, respectively, and the foot progression angles were $-15.67^{\circ}$ and $-17.99^{\circ}$, respectively, with neither of these differences being statistically significant. In contrast, on pAFO walking, the mean angles of hip rotation and foot progression were $-3.35^{\circ}$ and $-23.68^{\circ}$, respectively (Table 3 ). Both hip external rotation and external foot progression were significantly higher after wearing the pAFO $(\mathrm{p}<0.05)$ than on barefoot and HOAFO walking. While wearing pAFO, 10 subjects showed maximal external foot progression angle at the loading response. When we assessed the patient preference, we found eight preferred HOAFO, two preferred pAFO, and five reported little difference between them.

Table 2. Gait analysis: spatiotemporal parameters

\begin{tabular}{lllll}
\hline & Barefoot & \multicolumn{1}{c}{ pAFO } & \multicolumn{1}{c}{ HOAFO } & p-value \\
\hline Cadence (step/min) & $70.3(53.5,92.3)$ & $76.1(64.9,83.3)$ & $73.0(69.7,92.3)$ & 0.08 \\
Speed (cm/sec) & $34.0(22.5,60.1)$ & $40.1(30.3,51.8)$ & $39.0(35.5,53.7)^{\mathrm{b})}$ & $0.008^{\mathrm{a})}$ \\
Stride length (cm) & $59.7(48.0,73.0)$ & $65.4(58.4,77.5)$ & $65.6(58.2,71.5)$ & 0.06 \\
Step width (cm) & $14.4(13.8,18.1)$ & $16.5(14.3,19.3)$ & $16.3(14.8,18.0)$ & 0.07 \\
SLS (\%) & $27.0(18.5,32.6)$ & $27.5(23.5,33.5)$ & $28.3(23.1,30.5)^{\mathrm{b})}$ & $0.04^{\text {a) }}$ \\
DLS (\%) & $37.7(26.1,49.3)$ & $33.1(26.0,40.9)$ & $33.9(26.6,42.2)$ & 0.09 \\
\hline
\end{tabular}

Values are presented as median (interquartile range).

pAFO, plastic ankle-foot orthosis; HOAFO, heel-opened ankle-foot orthosis; SLS, single limb support; DLS, double limb support.

${ }^{a)} \mathrm{p}<0.05$ among three groups (repeated measures analysis of variance), ${ }^{b)} \mathrm{p}<0.05$ between BF and HOAFO in Tukey multiple comparison test.

Table 3. Gait analysis: kinematic data on the transverse plane during the stance phase

\begin{tabular}{lcccc}
\hline & Barefoot & pAFO & HOAFO & p-value \\
\hline Pelvis rotation $\left({ }^{\circ}\right)$ & $3.51(2.24)$ & $3.72(2.24)$ & $3.14(2.24)$ & 0.69 \\
Hip rotation $\left({ }^{\circ}\right)$ & $1.38(4.03)$ & $-3.35(4.03)$ & $0.78(4.03)$ & $<0.001^{\text {a) }}$ \\
Foot progression $\left(^{\circ}\right)$ & $-15.67(2.41)$ & $-23.68(2.41)$ & $-17.99(2.41)$ & $<0.001^{\text {a) }}$ \\
\hline
\end{tabular}

Values are presented as least squares means (standard error).

pAFO, plastic ankle-foot orthosis; HOAFO, heel-opened ankle-foot orthosis; negative (-), external rotation.

${ }^{a} \mathrm{p}<0.01$ among three groups (repeated measures analysis of variance). 


\section{DISCUSSION}

This study showed that, in hemiparetic stroke patients, the angle of foot external rotation of the affected limb was significantly lower while walking with HOAFO than with pAFO during the stance phase. Since hemiparetic stroke patients who already have pAFO showed excessive external foot progression during gait with $\mathrm{pAFO}$, heel opening of pAFO may be effective without increasing costs or shoe modification. Although there were studies using modified shoes to reduce foot external rotation, the shoes were either bulky or expensive $[12,13]$.

Gait pattern analysis comparing AFO with its absence is usually performed in the sagittal and frontal planes, with few studies reporting gait analysis on the transverse plane in patients with stroke. We were concerned about the excessive external foot rotation during the stance phase of gait in patients who wore pAFO. In a previous study, the hip was in a neutral position at its initial contact, with peak internal rotation occurring at the end of the loading response and peak external rotation at the end of pre-swing [18]. Another study showed that hip external rotation occurred at the terminal stance and remained externally rotated during the swing phase. Internal rotation of the hip occurred just before the initial contact, remaining until the terminal stance. These gait patterns were observed in most study subjects, with the average transverse rotation being $13^{\circ}[19]$. Electromyographic analysis showed that the hip abductor muscles, including the gluteus medius, upper gluteus maximus, and tensor fascia lata muscles, were activated during the initial half of the stance phase. Prolonged action of the semimembranosus during these phases maintained the thigh in an internally rotated position resisting the abductors [20]. In statistical results, we found that external rotation of both the hip and foot were increased in subjects who wore pAFO. However, when the degree of rotation was analyzed, external rotation of the foot was greater than the hip. This increase may have been due to the weakness of muscles of the affected limb and increased external rotation torque due to the solid plastic surface at the heel of pAFO during the loading response by decreasing frictional force.

Pelvic movement during gait is another important factor associated with energy efficiency and normal walking [21]. The pelvic rotation angle during comfortable walk- ing has been reported to be about $11.5^{\circ}$ in healthy subjects, but was decreased in elderly subjects. Decreased pelvic rotation may decrease stride length and gait speed in elderly individuals [22]. Studies of pelvic rotation in stroke patients, however, have not shown consistent results $[23,24]$. In one study, pelvic rotation in stroke patients did not differ from that in the normal control subjects, and could be larger or smaller, suggesting that pelvic rotation was not an appropriate tool for evaluating gait pattern in stroke patients [23]. Another study found that stroke patients who had slow walking speed showed pelvic retraction (external rotation) during the stance phase [24]. In the present study, we found that external pelvic rotation during the stance phase was uncertain among the three conditions of walking. Pelvic rotation may have affected the speed and efficiency of gait, but wearing pAFO or HOAFO did not affect pelvic movement of the affected limb in our study. The mechanism underlying this phenomenon warrants further investigation in the assessment of pathologic gait after hemiplegic stroke.

About $70 \%-80 \%$ of stroke patients can walk short distances independently, but only about $50 \%$ can walk longer distances; moreover, most of these patients have decreased gait speed, stride length, and stability and increased energy expenditure $[25,26]$. Wearing pAFO is frequently used to increase gait stability and improve walking function. Our results did not show that wearing pAFO resulted in statistically significant improvements; although, most parameters were generally better than those observed during barefoot walking. Each of our study subjects walked comfortably, but not at maximal speed, during gait analysis. Spatiotemporal parameters showed that wearing HOAFO also improved all parameters, with gait speed and percentage of SLS being significantly improved compared with barefoot walking. These findings indicate that HOAFO may provide additional effects on gait, compared with pAFO, by decreasing external rotation of the hip and foot during stance phase; thereby, improving gait function in patients with hemiplegic stroke.

This study had several limitations. First, the relatively small sample size may have contributed to the lack of significant differences in spatiotemporal parameters and kinematic data. Second, we evaluated only the affected limb and kinematic data on the transverse plane. The angle measured in the transverse plane may have more er- 
rors than the values of frontal or sagittal plane, but most subjects of our study showed consistent results. Third, some subjects were not well fitted to these orthoses because only three sizes of pAFOs and HOAFO were available. Fourth, the gait analysis was not performed wearing shoes. Finally, despite the 3-dimensional gait analysis that resulted in improvements in the external progression of foot angle during stance phase after wearing HOAFO, we did not assess the exact mechanism of these improvements. Therefore, additional studies are needed to clarify these mechanisms and to develop other methods to reduce this phenomenon.

In conclusion, we found that HOAFO was superior to pAFO for reducing hip and foot external rotation during the stance phase of the gait cycle in patients with poststroke hemiparesis. In addition, HOAFO significantly improved gait speed and the percentage of SLS compared with that of barefoot walking. HOAFO may, therefore, be useful for patients with excessive external rotation of the foot during the use of a conventional pAFO and may be created by cutting the calcaneal area of the latter.

\section{CONFLICT OF INTEREST}

No potential conflict of interest relevant to this article was reported.

\section{REFERENCES}

1. da Cunha-Filho IT, Henson H, Wankadia S, Protas EJ. Reliability of measures of gait performance and oxygen consumption with stroke survivors. J Rehabil Res Dev 2003;40:19-25.

2. Olney SJ, Monga TN, Costigan PA. Mechanical energy of walking of stroke patients. Arch Phys Med Rehabil 1986;67:92-8.

3. Kim CM, Eng JJ. Magnitude and pattern of 3D kinematic and kinetic gait profiles in persons with stroke: relationship to walking speed. Gait Posture 2004;20:140-6.

4. Cruz TH, Dhaher YY. Impact of ankle-foot-orthosis on frontal plane behaviors post-stroke. Gait Posture 2009;30:312-6.

5. de Wit DC, Buurke JH, Nijlant JM, Ijzerman MJ, Hermens HJ. The effect of an ankle-foot orthosis on walking ability in chronic stroke patients: a randomized controlled trial. Clin Rehabil 2004;18:550-7.

6. Embrey DG, Holtz SL, Alon G, Brandsma BA, McCoy SW. Functional electrical stimulation to dorsiflexors and plantar flexors during gait to improve walking in adults with chronic hemiplegia. Arch Phys Med Rehabil 2010;91:687-96.

7. Sheffler LR, Hennessey MT, Naples GG, Chae J. Peroneal nerve stimulation versus an ankle foot orthosis for correction of footdrop in stroke: impact on functional ambulation. Neurorehabil Neural Repair 2006; 20:355-60.

8. Cakar E, Durmus O, Tekin L, Dincer U, Kiralp MZ. The ankle-foot orthosis improves balance and reduces fall risk of chronic spastic hemiparetic patients. Eur J Phys Rehabil Med 2010;46:363-8.

9. Gok H, Kucukdeveci A, Altinkaynak H, Yavuzer G, Ergin S. Effects of ankle-foot orthoses on hemiparetic gait. Clin Rehabil 2003;17:137-9.

10. Chen CC, Hong WH, Wang CM, Chen CK, Wu KP, Kang CF, et al. Kinematic features of rear-foot motion using anterior and posterior ankle-foot orthoses in stroke patients with hemiplegic gait. Arch Phys Med Rehabil 2010;91:1862-8.

11. Roth EJ, Merbitz C, Mroczek K, Dugan SA, Suh WW. Hemiplegic gait: relationships between walking speed and other temporal parameters. Am J Phys Med Rehabil 1997;76:128-33.

12. Joo BG, Yoo JY, Ha SB. Effect of torque heel on excessive external rotation of hemiplegic foot: three dimensional gait analysis. J Korean Acad Rehabil Med 1998;22:1114-22.

13. Lee JK, Lim OK, Yim YM, Chung SR, Bae KH, Kim SH, et al. The shoes designed to inhibit excessive external rotation of hemiplegic foot with plastic ankle foot orthosis: a case report. J Korean Acad Rehabil Med 2006;30:94-7.

14. Bohannon RW, Smith MB. Interrater reliability of a Modified Ashworth Scale of muscle spasticity. Phys Ther 1987;67:206-7.

15. Miyamoto S, Kondo T, Suzukamo Y, Michimata A, Izumi S. Reliability and validity of the Manual Function Test in patients with stroke. Am J Phys Med Rehabil 2009;88:247-55.

16. Cameron D, Bohannon RW. Criterion validity of lower extremity Motricity Index scores. Clin Rehabil 2000;14: 208-11. 
17. Blum L, Korner-Bitensky N. Usefulness of the Berg Balance Scale in stroke rehabilitation: a systematic review. Phys Ther 2008;88:559-66.

18. Levens AS, Inman VT, Blosser JA. Transverse rotation of the segments of the lower extremity in locomotion. J Bone Joint Surg Am 1948;30A:859-72.

19. Johnston RC, Smidt GL. Measurement of hip-joint motion during walking: evaluation of an electrogoniometric method. J Bone Joint Surg Am 1969;51:1082-94.

20. Lyons K, Perry J, Gronley JK, Barnes L, Antonelli D. Timing and relative intensity of hip extensor and abductor muscle action during level and stair ambulation: an EMG study. Phys Ther 1983;63:1597-605.

21. Saunders JB, Inman VT, Eberhart HD. The major determinants in normal and pathological gait. J Bone
Joint Surg Am 1953;35A:543-58.

22. Murray MP. Gait as a total pattern of movement. Am J Phys Med 1967;46:290-333.

23. Wagenaar RC, Beek WJ. Hemiplegic gait: a kinematic analysis using walking speed as a basis. J Biomech 1992;25:1007-15.

24. De Quervain IA, Simon SR, Leurgans S, Pease WS, McAllister D. Gait pattern in the early recovery period after stroke. J Bone Joint Surg Am 1996;78:1506-14.

25. Jorgensen HS, Nakayama H, Raaschou HO, Olsen TS. Recovery of walking function in stroke patients: the Copenhagen Stroke Study. Arch Phys Med Rehabil 1995;76:27-32.

26. Friedman PJ. Gait recovery after hemiplegic stroke. Int Disabil Stud 1990;12:119-22. 